\title{
Seismic imaging of hydraulically-stimulated fractures: A numerical study of the effect of the source mechanism
}

Andrey H. Shabelansky*, Alison Malcolm, Mike Fehler, and Sudhish K. Bakku, Earth Resources Laboratory, Massachusetts Institute of Technology

\section{SUMMARY}

We present a numerical study of seismic imaging of hydraulically stimulated fractures using a single source from an adjacent fracturing-process. The source is either a point force generated from the perforation of the casing of the well or a double-couple as is typically observed from the induced microseismicity. We assume that the fracture is sufficiently stimulated to be imaged by reflected seismic energy. We show for a specific monitoring geometry of hydrofracturing that not only different waves (P and $\mathrm{S}$ ) but also different source mechanisms from the same region form an image of different parts of the target fracture and thus add complementary information. The strategy presented here might be used as an additional monitoring tool of the hydrofracturing process.

\section{INTRODUCTION}

In recent years hydraulic fracturing has become a key driver for the economic production of unconventional low permeability reservoirs. Monitoring hydraulic fracturing is important because it helps optimize the stimulation process and in assessing the hazards which might be generated from induced seismicity. Conventional monitoring techniques involve methods of hypocenter event relocation (Rutledge and Phillips, 2003), moment tensor inversion for source mechanism (Nolen-Hoeksema and Ruff, 1999; Song and Toksoz, 2011), and travel time tomography for velocity and anisotropy parameter estimation (Idelton et al., 1993). Neither of those techniques address the explicit imaging of the fractures in the vicinity of the stimulation. Nevertheless, information recovered by such techniques is crucial and necessary for reliable imaging. Two types of sources are generally present in the hydraulic stimulation process. The first is generated by the perforation of the casing of the well (Warpinski et al., 2003) and presumably radiates most of its energy as a point force monopole. Second is the induced microseismicity which generally has a dominant double couple mechanism (Song et al., 2010; Song and Toksoz, 2011). Sources with the second mechanism are numerous but often weak and radiate stronger S-waves than P-waves (Song et al., 2010). However, S-waves monitored on the surface are generally attenuated by the near surface (Duncan and Eisner, 2010). Therefore, imaging with S-waves using monitoring wells seems to be the most promising option, despite problems specific to downhole monitoring such as tube waves and receiver coupling (Eisner et al., 2011). Imaging of fractures using S-waves has two additional advantages. First, the wavelength of S-wave is shorter that that of the P-wave allowing better imaging resolution. Second, not only the source mechanism but also the tangential and normal compliances of the fractures are more sensitive to S-waves (Lubbe et al., 2008; Gurevich et al., 2009; Fang et al., 2010).
In this paper we outline an approach for imaging of stimulated fractures using sources from successive adjacent stimulations. We numerically show the effect of the source mechanism on the imaging by constructing four types of images (PP, PS, SP, SS) for data generated by sources with the two different source mechanisms mentioned above: point force monopole and double couple. We also test the effect of the orientation of the double couple source. The results are interpreted using the reflection coefficients analytically derived for our simplified model of a hydraulically-stimulated fracture.

\section{IMAGING STRATEGY}

Our imaging strategy uses the recorded seismic signals from the fracturing-process similarly to those recorded from the seismic while drilling (SWD) (Rector and Marion, 1991), however instead of using the drill-bit as a source we use either the perforation source or induced-seismicity source. For the latter source we assume that the relocation and moment tensor inversions are performed prior to the imaging. We also assume that the stimulated fracture which we wish to image is quasi perpendicular to the treatment well because of the continuos controlled stimulation that the fracture undergoes. In our study we position the treatment and the monitoring wells vertically (see figure 1), however the strategy can be applied similarly for horizontal wells and is not restricted to a single monitoring well. For the perforation source we assume that the radiation energy pattern is a point force monopole in the horizontal direction (see top left corner in figure 1), and for the microseismic source the mechanism is double-couple (bottom left corner in figure 1). Although both source mechanisms radiate both $\mathrm{P}$ - and $\mathrm{S}$-waves, there is significantly more S-wave energy.

To make use of the full waveform information for imaging using a single shot, we use the reverse time migration (RTM) algorithm (Baysal et al., 1983) using an elastic framework. The imaging condition for the elastic case is the same as in Claerbout (1971) adapted for the elastic case similarly to Sun and McMechan (1986) as

$$
I_{\gamma \eta}(\mathbf{x})=\int_{0}^{T} U_{\gamma}^{f}(\mathbf{x}, t) U_{\eta}^{b}(\mathbf{x}, T-t) d t
$$

where $\mathbf{x}$ is spatial position, $t$ is time, $T$ is the maximum recorded time, and superscripts $f$ and $b$ denote the forward and backward propagation, respectively. The wavefield $U$ denotes the particle velocity wavefield where subscripts $\gamma$ and $\eta$ each refers to the following wavefields: $X, Z, P, S$, where $X$ and $Z$ are the horizontal and vertical components, and $P$ and $S$ are P- and vertically polarized $S$-waves, respectively. Note that although in our study we use $U$ as the particle-velocity, the acceleration 


\section{Microseismic imaging using different source mechanisms}

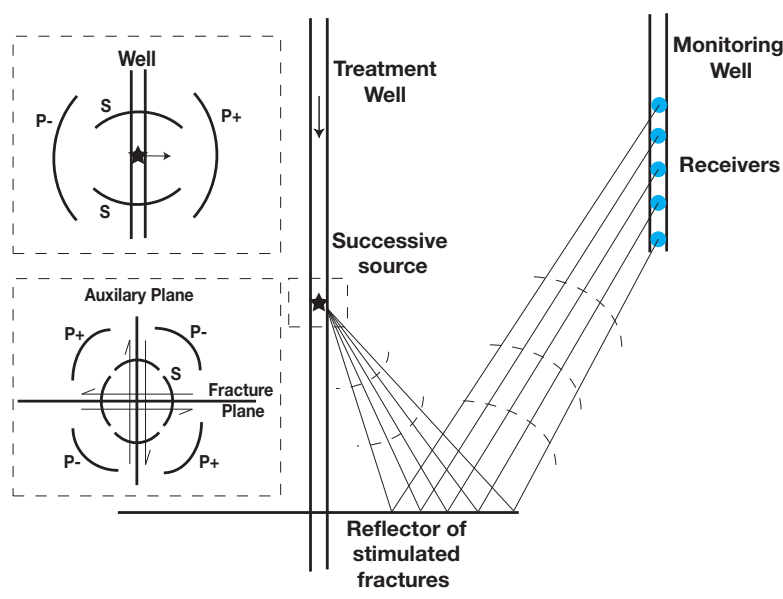

Figure 1: Schematic showing the geometry of the seismic imaging of the fractured reflector using a source. The box in the top left corner shows the horizontal point force (monopole) mechanism of the perforation source, and the box below shows the double couple source generated from the induced seismicity. Note that even though the fracture plane of the doublecouple source is drawn horizontally, it can be tilted in any direction, accordingly changing the radiation energy pattern of $\mathrm{P}$ and $\mathrm{S}$ waves.

field, occasionally recorded in the hydrofracturing monitoring (Warpinski et al., 1998), can be used similarly.

Before showing the numerical results of the elastic imaging, we calculate the analytical elastic reflection coefficients as a function of the incident angle of two half spaces. We use this as a model of a fracture, assuming that the shear modulus change is the dominant perturbation caused by the stimulation. We use the approximate formulas given in Aki and Richards (2002) (p. 148) with Lame parameters. Figure 2 shows the reflected coefficients for PP, PS, SP, SS wave modes, where the first and the second letters refer to the incident and the reflected modes, respectively. We used homogeneous background Lame parameter $\lambda$ of $15.5 \mathrm{GPa}$, shear modulus, $\mu$, of $12.5 \mathrm{GPa}$, and density, $\rho$, of $2000 \mathrm{~kg} / \mathrm{m}^{3}$ perturbing only shear modulus with $-5 \%,-10 \%,-15 \%,-20 \%,-25 \%$, and $-30 \%$ of the background value in the second layer. The reason for perturbing only shear modulus stems from observations suggesting that the tangential compliance (inverse of the shear modulus) is more sensitive to changes than the normal compliance (inverse of the bulk modulus) (Lubbe et al., 2008; Gurevich et al., 2009; Fang et al., 2010), although in general other physical parameters can be affected as well. We observe that the reflectivity of the PP mode (figure $2 \mathrm{a}$ ) is positive at zero angle and decreases to zero with increasing incident angle after which it increases again; there is no change in polarity. In contrast, the other modes have a negative reflection coefficient at zero angle and decrease (in absolute sense) until they change polarity (see the dotted lines in figure 2). Note that the converted modes PS and SP (figures $2 \mathrm{~b}$ and c) change polarity at larger angles than the SS mode indicating that imaging with those modes may provide an image over a larger region than that with SS mode (figure $2 \mathrm{~d}$ ). The short distance between the wells may still allow the use of the SS mode however. In figure 3 we also show the potential paths of each of the wave modes between a single source in the well, the fractured reflector, and the nearest receiver in the monitoring well. We show that using the fact that the incident and the reflected angles of P-waves are always larger than those of S-waves, the path of the PS mode samples the farthest points on the reflector better than the nearest points, the SP mode samples the nearest points better than the farthest, and the PP and SS modes sample the intermediate points.
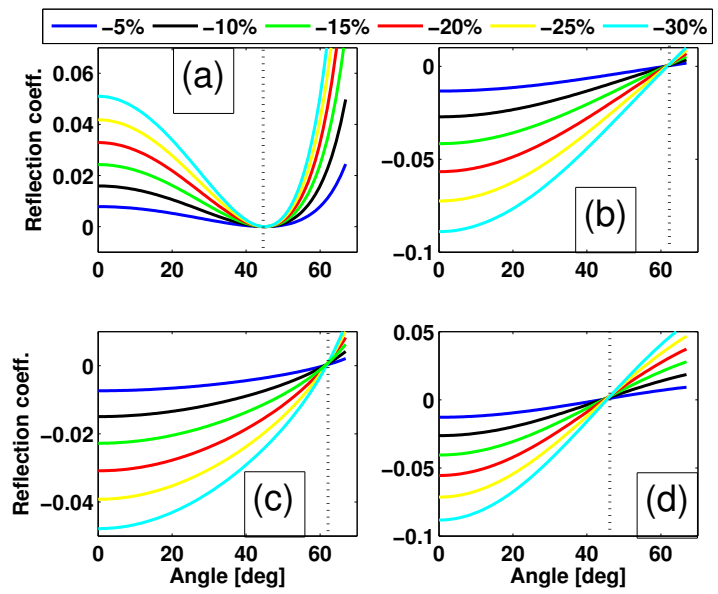

Figure 2: Reflection coefficients as a function of the incident angle with Lame parameters $\lambda=15.5 \mathrm{GPa}, \mu=12.5 \mathrm{GPa}, \rho=$ $2000 \mathrm{~kg} / \mathrm{m}^{3}$, and different $\Delta \mu$ (the percentage of the change listed at the top of the figure) for different wave modes: (a) PP, (b) PS, (c) SP, and (d) SS. The dotted vertical lines mark to the angle where the wave modes change polarity.

\section{SYNTHETIC TESTS}

We test the proposed imaging strategy with three 2D examples. In the first example we use the horizontal point force source mechanism, in the second the double-couple with horizontalvertical nodal-auxilary planes, and in the third example with the double-couple mechanism with the planes rotated $45^{\circ}$ from the horizontal-vertical axes. We choose an angle of $45^{\circ}$ to sample the other extreme in the radiation pattern, which could be at any angle. All examples were performed with a simple twolayer model representing the overburden and a layer containing the stimulated fractures at a depth of $150 \mathrm{~m}$ (see figure 3). We use the same $\lambda=15.5 \mathrm{GPa}, \mu=12.5 \mathrm{GPa}, \rho=2000 \mathrm{~kg} / \mathrm{m}^{3}$, with which we estimated the reflection coefficients in figure 2 , using $\Delta \mu=-20 \%$. For each example we generate a single shot in the treatment well at the horizontal position of $140 \mathrm{~m}$ and the depth of $102 \mathrm{~m}$ using a Ricker wavelet with the typically observed peak frequency of $250 \mathrm{~Hz}$ (Song and Toksoz, 2011). The time step is $0.04 \mathrm{~ms}$ and the maximum recorded time is $0.15 \mathrm{~s}$. The recording receivers are located at a distance of $100 \mathrm{~m}$ at the horizontal position of $240 \mathrm{~m}$ and vertical depths between 40 and $100 \mathrm{~m}$ separated with $4 \mathrm{~m}$ (Nolen-Hoeksema 


\section{Microseismic imaging using different source mechanisms}

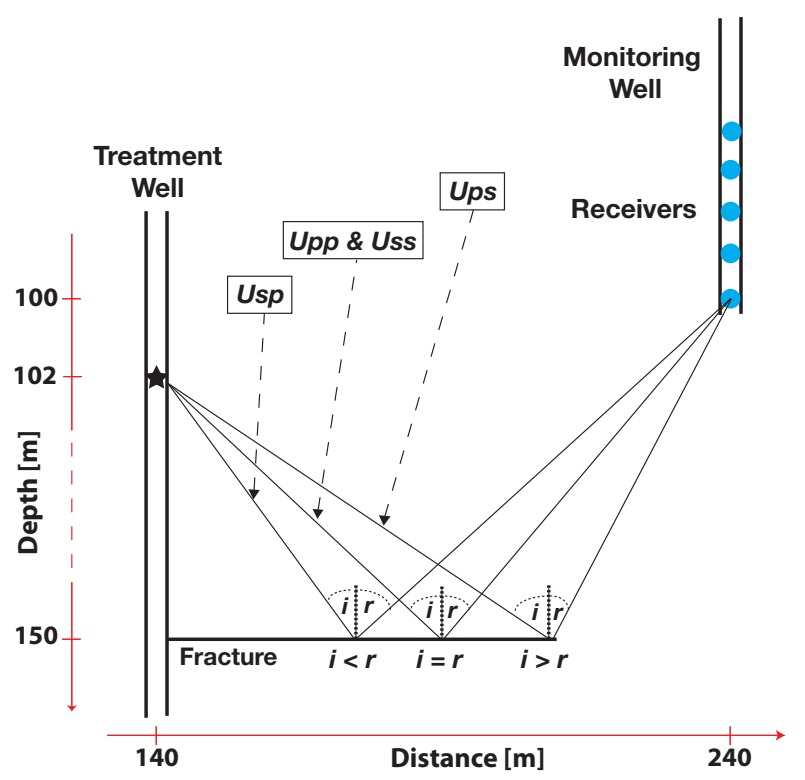

Figure 3: General ray paths of the different wave modes $\left(U_{P P}\right.$, $\left.U_{P S}, U_{S P}, U_{S S}\right)$ showing the relationship between the source, reflector, and the nearest receiver and their corresponding incident $(i)$ and reflection $(r)$ angles.

and Ruff, 1999; Song and Toksoz, 2011). All synthetic data are modeled with 2D full elastic velocity-stress staggered-grid finite difference solver using a second order in time pseudospectral method with perfectly matched layer (PML) boundary conditions (Marcinkovich and Olsen, 2003; Carcione, 1999). The incorporation of the double-couple source mechanism follows Wu and Maupin (2007), (p. 481-483). The wavefields $U_{P}$ and $U_{S}$ are calculated from the divergence and the curl of the particle-velocity wavefields, respectively. All images presented below are filtered with a Laplacian filter (Youn and Zhou, 2001) to remove the low frequency artifacts typical for the RTM.

In figures 4 (a)-(l) we show images zoomed around the reflector that were obtained with a single shot using the elastic RTM from the PP, PS, SP, and SS waves, where the source mechanism is the single horizontal force (monopole) generated from vertical treatment well (figures 4 (a)-(d)), the double couple along horizontal-vertical axes (figures 4 (e)-(h)), and the double couple rotated by 45 degrees from the horizontalvertical axes (figures 4 (i)-(l)). We observe that all PP images have poor resolution regardless of the source mechanism as expected due to the larger wavelength of P-waves. In the PS images, we observe that different parts of the fractured reflector are imaged by different source mechanisms suggesting that events with different source mechanism even from the same location image different regions of the reflector as long as sufficient receiver coverage exists, and as long as the polarity change does not occur. Here, the maximum opening angle is about $45^{\circ}$ which results in no change in the amplitude polarity for all modes (see figure 2). For SP modes we observe that only those parts of the reflector that are close to the treatment well are imaged despite their similar angle range with the PS modes in figure 2. For SS wave modes we observe that the images are radiation pattern dependent as with the PS modes, suggesting that these two modes are the most sensitive to the source mechanism. We also observe that the SS modes are free of artifacts unlike the other wave modes. These artifacts (marked with the black arrows in figure 4) are caused by the SP conversion at the interface during the RTM propagation (Deng and McMechan, 2008).

Since microseismic data is weak and P-waves are often below the noise level (Song et al., 2010), it is difficult to obtain reliable images with a single source. To increase the signal to noise ratio of the images and because we observed that different source mechanisms image different parts of the fractured layer, we stack the images over the three different source mechanisms (see figures $4(\mathrm{~m})$-(p)). These results show that the signal to noise ratio of the images, particularly of the PS mode, is improved by stacking despite the limited receiver coverage. Because induced seismicity generates sources at different locations around the treatment well, we also stack the images over eight separate sources locations, which are located at the same depth of $102 \mathrm{~m}$ and horizontal distances between $110 \mathrm{~m}$ and $180 \mathrm{~m}$ with $10 \mathrm{~m}$ spacing. Figures $4(\mathrm{~m})-(\mathrm{p})$ show these results for the stacked over all 24 shots. We observe that stacking over the source locations reduced the artifacts caused by the SP converted phase.

\section{CONCLUSIONS}

We presented a numerical study of imaging of the hydraulicallystimulated fractures using a successive single source with different source mechanisms: point force monopole, horizontalvertical double couple, rotated by $45^{\circ}$ double couple. We observed that the PS wave mode gives the best image of the fracture with the treatment-monitoring geometry. We also observed that the orientation of the source mechanism significantly affects the seismic imaging which suggests that microseismic events from the similar locations with different source mechanism can constructively image different parts of the target. These results suggest that imaging one fracture with microseismic sources in another may be possible even with small contrasts and noisy data.

\section{ACKNOWLEDGMENTS}

We wish to thank the sponsors of the Earth Resources Laboratory at MIT for their support. We wish also to thank M. Nafi Toksoz, Yves Bernabe, Xinding Fang, Junlun Li, and Yingcai Zheng for their helpful discussions. 


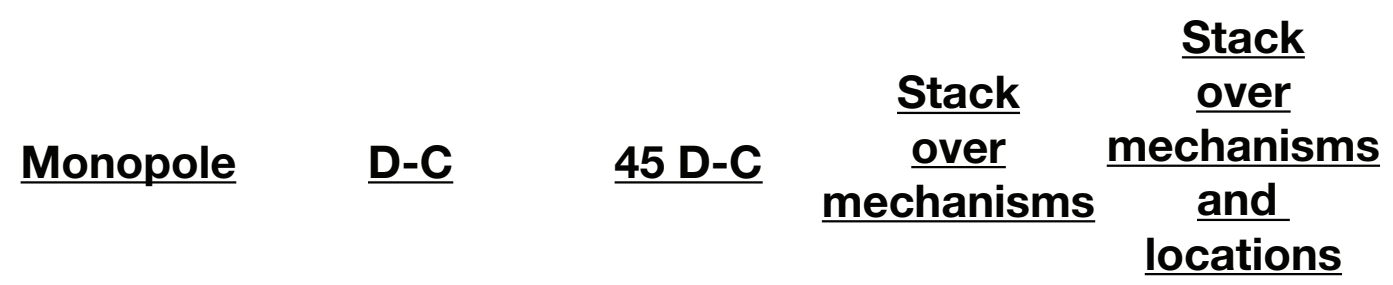

(a)

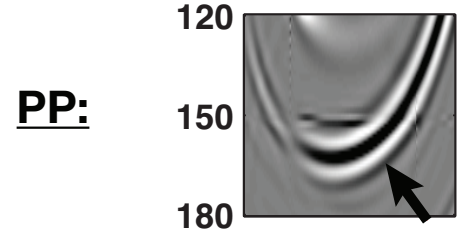

(b)

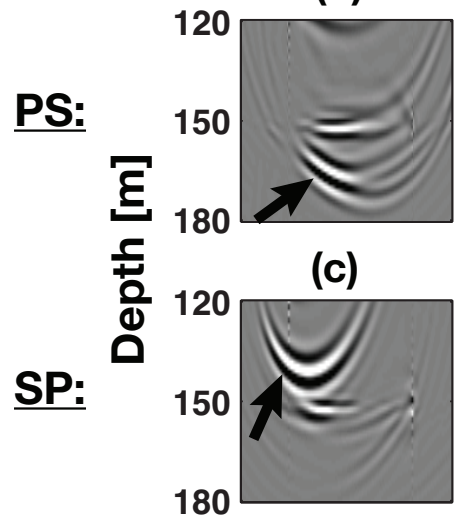

(d)

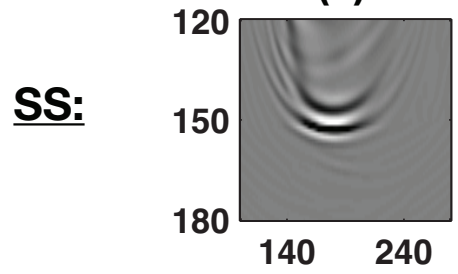

(e)

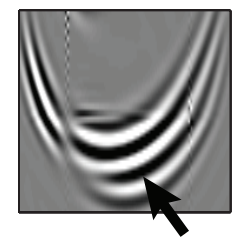

(f)

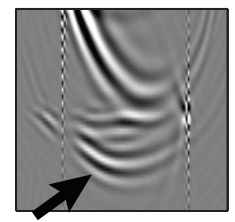

(g)

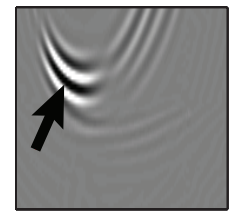

(h)

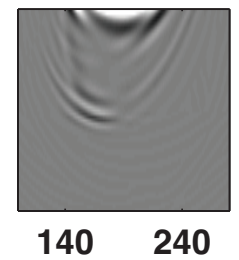

(i)

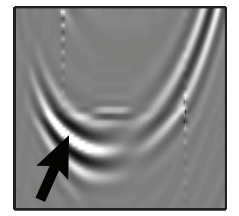

(j)

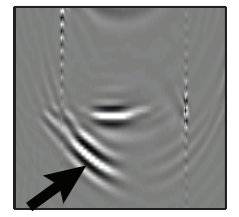

(k)

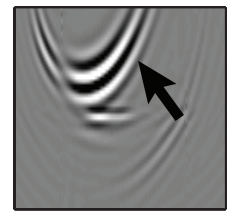

(l)

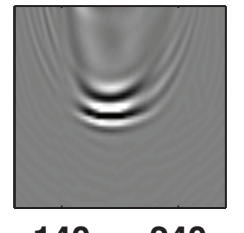

$140 \quad 240$ (m)

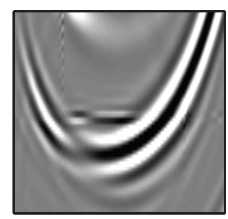

(n)

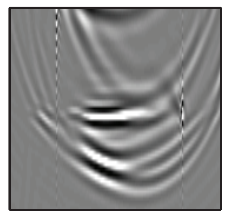

(o)

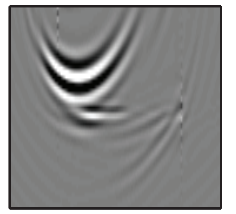

(p)

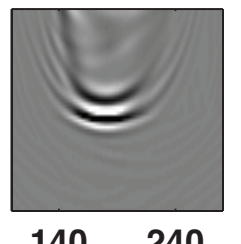

$140 \quad 240$ (q)

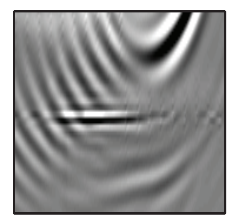

(r)

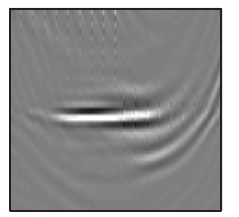

(s)

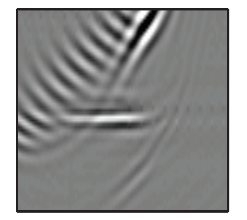

(t)

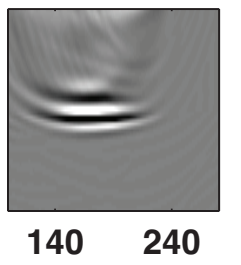

Distance [m]

\section{$-0.6$}

0.0

0.6

Figure 4: Elastic RTM images zoomed around the reflector that were obtained by migration with different source mechanisms where each row corresponds to the image with each of the following wave modes: PP, PS, SP, SS, respectively, generated with (a)-(d) horizontal point force monopole (e)-(h) horizontal-vertical double couple, and (i)-(l) $45^{\circ}$ rotated double couple source mechanisms, (m)-(p) images obtained from stacking of the images of the three mechanisms, (q)-(t) images obtained from stacking over three source mechanisms and eight locations at the depth of $102 \mathrm{~m}$ and horizontal distance between $100 \mathrm{~m}$ and $180 \mathrm{~m}$ separated with $10 \mathrm{~m}$ (total 24 sources). The black arrows mark the artifacts caused by the SP converted phases. 
http://dx.doi.org/10.1190/segam2012-1182.1

\section{EDITED REFERENCES}

Note: This reference list is a copy-edited version of the reference list submitted by the author. Reference lists for the 2012 SEG Technical Program Expanded Abstracts have been copy edited so that references provided with the online metadata for each paper will achieve a high degree of linking to cited sources that appear on the Web.

\section{REFERENCES}

Aki, K., and P. G. Richards, 2002, Quantitative seismology: Theory and Methods, 2 ed.: University Science Books

Baysal, E., D. Kosloff, and J. W. C. Sherwood, 1983, Reverse-time migration: Geophysics, 48, 15141524.

Carcione, J. M., 1999, Staggered mesh for the anisotropic and viscoelastic wave equation: Geophysics, 64, 1863-1866.

Claerbout, J. F., 1971, Toward a unified theory of reflector mapping: Geophysics, 36, 467-481.

Deng, F., and G. A. McMechan, 2008, Viscoelastic true-amplitude prestack reverse-time depth migration: Geophysics, 73, no. 4, S143-S155.

Duncan, P., and L. Eisner, 2010, Reservoir characterization using surface microseismic monitoring: Geophysics, 75, no. 5, 75A139-75A146.

Eisner, L., M. Thornton, and J. Griffin, 2011, Challenges for microseismic monitoring: $81^{\text {st }}$ Annual International Meeting, SEG, Expanded Abstracts, 1519-1523.

Fang, X., M. Fehler, T. Chen, and D. Burns, 2010, Sensitivity analysis of fracture scattering: Presented at the 80th Annual International Meeting, SEG.

Gurevich, B., D. Makarynska, and M. Pervukhina, 2009, Are penny-shaped cracks a good model for compliant porosity?: 79th Annual International Meeting, SEG, Expanded Abstracts, 3431-3435.

Idelton, D., T. Patzek, L. Rector, and H. Vinegar, 1993, Microseismic imaging of hydrofractures with aid of conical waves: 63rd Annual International Meeting, SEG, Expanded Abstracts, 267-270.

Lubbe, R., J. Sothcott, M. Worthington, and C. McCann, 2008, Laboratory estimates of normal and shear fracture compliance: Geophysical Prospecting, 56, 239-247.

Marcinkovich, C., and K. Olsen, 2003, On the implementation of perfectly matched layers in a threedimensional fourth-order velocity-stress finite difference scheme: Journal of Geophysical Research, 108, no. 18, 1-16.

Nolen-Hoeksema, R., and L. Ruff, 1999, Moment tensor inversion of microseismic events from hydrofractures: Presented at the 69th Annual International Meeting, SEG.

Rector, J., and B. Marion, 1991, The use of drill-bit energy as a downhole seismic source: Geophysics, 56, 628-634.

Rutledge, J., and W. Phillips, 2003, Hydraulic stimulations of natural fracture as revealed by induced microearthquakes, Carthage Cotton Valley gas field, east Texas: Geophysics, 68, 441-452.

Song, F., H. S. Kuleli, M. Toksoz, E. Ay, and H. Zhang, 2010, An improved method for hydrofractureinduced microseismic event detection and phase picking: Geophysics, 75, no. 6, A47-A52. 
Song, F., and M. Toksoz, 2011, Full-waveform based complete moment tensor inversion and source parameter estimation from downhole microseismic data for hydrofracture monitoring: Geophysics, 76, no. 6, WC101-WC114.

Sun, R., and G. McMechan, 1986, Prestack reverse-time migration for elastic waves with application to synthetic offset vertical seismic profiles: Proceeding of the IEEE, 74, 457-465.

Warpinski, N., P. Branagan, R. Peterson, S. Wolhart, and J. Uhl, 1998, Mapping hydraulic fracture growth and geometry using microeseismic events detected by a wireline retrievable accelerometer array: SPE.

Warpinski, N., R. Sullivan, J. Uhl, C. Waltman, and S. Machovoe, 2003, Improved microseismic fracture mapping using perforation timing measurements for velocity calibration: SPE.

Wu, R.-S., and V. Maupin, 2007, Advances in geophysics: Advances in wave propagation in heterogeneous earth, 2 ed.: Elsevier.

Youn, O., and H.-W. Zhou, 2001, Depth imaging with multiples: Geophysics, 66, 246-255. 